\title{
Succession of the turkey gastrointestinal bacterial microbiome related to weight gain
}

Because of concerns related to the use of antibiotics in animal agriculture, antibiotic-free alternatives are greatly needed to prevent disease and promote animal growth. One of the current challenges facing commercial turkey production in Minnesota is difficulty obtaining flock average weights typical of the industry standard, and this condition has been coined "Light Turkey Syndrome" or LTS. This condition has been identified in Minnesota turkey flocks for at least five years, and it has been observed that average flock body weights never approach their genetic potential. However, a single causative agent responsible for these weight reductions has not been identified despite numerous efforts to do so. The purpose of this study was to identify the bacterial community composition within the small intestines of heavy and light turkey flocks using $16 \mathrm{~S}$ rRNA sequencing, and to identify possible correlations between microbiome and average flock weight. This study also sought to define the temporal succession of bacteria occurring in the turkey ileum. Based upon 2.7 million sequences across nine different turkey flocks, dominant operational taxonomic units (OTUs) were identified and compared between the flocks studied. OTUs that were associated with heavier weight flocks included those with similarity to Candidatus division Arthromitus and Clostridium bartlettii, while these flocks had decreased counts of several Lactobacillus species compared to lighter weight flocks. The core bacterial microbiome succession in commercial turkeys was also defined. Several defining markers of microbiome succession were identified, including the presence or abundance of Candidatus division Arthromitus, Lactobacillus aviarius, Lactobacillus ingluviei, Lactobacillus salivarius, and Clostridium bartlettii. Overall, the succession of the ileum bacterial microbiome in commercial turkeys proceeds in a predictable manner. Efforts to prevent disease and promote growth in the absence of antibiotics could involve target dominant bacteria identified in the turkey ileum that are associated with increased weight gain. 
1 Jessica L. Danzeisen ${ }^{1}$, Alamanda J. Calvert ${ }^{2}$, Sally L. Noll², Brian McComb ${ }^{3}$, Julie S. Sherwood ${ }^{4}$,

2 Catherine M. Logue ${ }^{4,5}$, and Timothy J. Johnson ${ }^{1 *}$

$3{ }^{1}$ Department of Veterinary and Biomedical Sciences, College of Veterinary Medicine, University 4 of Minnesota, Saint Paul, MN 55108

$5{ }^{2}$ Department of Animal Sciences, College of Food, Agriculture and Natural Resource Sciences,

6 University of Minnesota, Saint Paul, MN 55108

$7 \quad{ }^{3}$ Willmar Poultry Company, Willmar, MN

$8{ }^{4}$ Department of Veterinary and Microbiological Sciences, North Dakota State University, Fargo, 9 ND, 58103

$10{ }^{5}$ Department of Veterinary Microbiology and Preventive Medicine, College of Veterinary

11 Medicine, Iowa State University, Ames, IA, 50011

$12 *$ Corresponding author. Department of Veterinary and Biomedical Sciences, College of

13 Veterinary Medicine, University of Minnesota, 301B Veterinary Science Building, 1971

14 Commonwealth Avenue, Saint Paul, MN 55108

15 Phone: 612.626.2542, Fax: 612.625.5203, Email: joh04207@umn.edu 
Introduction

The United States produces more than 250 million turkeys per year (USDA 2012),

18 resulting in a multi-billion dollar per year industry (USDA 2007). Growth performance and

19 sustained flock health is of major economic importance to commercial turkey producers. The microbial community of the gastrointestinal tract, or microbiome, is assumed to play a critical

21 role in overall health of turkeys and other poultry, but the composition of bacterial species within

22 the turkey gastrointestinal tract is largely understudied. Furthermore, the role of these microbes in

23 the development of a healthy intestinal tract is not entirely understood.

The onset of next-generation sequencing has resulted in a marked increase in cultureindependent studies characterizing the gut microbiome, but much of this work has been focused on humans and other production animals (Danzeisen et al. 2011; Kim et al. 2011; $\underline{\text { Scalzo et al. }}$ 2004). Fewer studies have sought to understand the turkey microbiome. Some work has focused

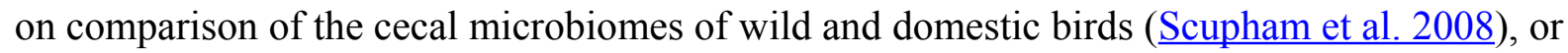
examination of the turkey microbiome in relation to pathogen colonization, such as

31 present in the ceca of differing types of turkeys, as well as time-dependent shifts in bacterial 32 populations in the turkey intestinal tract. However, such previous turkey studies relied on culture-

33 based methods or lower output molecular fingerprinting methods, such as T-RFLP ( $\underline{\text { Scupham }}$

34 2009; Scupham et al. 2008). These methods present limitations and biases that are not encountered with high-throughput sequencing (Handelsman 2004), which can be targeted at loci such as the 16S rRNA gene to achieve deep taxonomic coverage and better resolution. 
40 Growers Association). Lower than expected market weights associated with LTS seem to be most

41 prevalent in heavy tom flocks, since the problem is likely amplified over the longer growth

42 period of toms versus hens (Calvert 2012). LTS has been identified in Minnesota turkey flocks

43 for at least five years, and it has been observed that average flock body weights never approach

44 their genetic potential. The gap between observed weights and genetic potential starts

45 immediately at brooding and continues throughout the grow-out phase of the turkey (Calvert

46 2012). A number of possible contributing factors have been speculated, including management

47 practices, the presence of known or unknown bacterial or viral pathogens, disruptions of the

48 gastrointestinal microbial communities, problems with nutrient absorption, or dwarfed immune

49 development in poults (Calvert 2012). This problem has not, to our knowledge, been identified in

50 other states in the USA.

It is unclear if LTS is tied to another condition in turkeys called poult enteritis syndrome,

52 or PES (indal et al. 2009). Studies have demonstrated that inoculation of healthy birds with fecal

53 slurries from birds experiencing PES results in significant reductions in body weight compared to

54 controls (Mor et al. 2011). However, a single causative agent responsible for these weight

55 reductions has not been identified. Astroviruses, rotaviruses, and reoviruses have been found in

56 birds experiencing PES, but they are also found in apparently healthy birds (Jindal et al. 2012) or

57 studies examining these viruses lacked negative control groups (Jindal et al. 2010). LTS has been

58 reproduce in controlled animal studies using pooled fecal homogenates, but not individual

59 microogranisms (Mor et al. 2013). Also, no epidemiological associations have been made

60 between the PES and LTS, and LTS often occurs in the absence of PES. Overall, there is no

61 evidence to suggest a single pathogen associated with LTS.

62 The use of subtherapeutic concentrations of antimicrobial agents in animal agriculture

63 (i.e., growth promoters) is being increasingly scrutinized because of the rise of multidrug 
64 resistant pathogens. Therefore, there is an urgent need to identify antibiotic-free alternatives to

65 improving animal health and weight gain. If there is a microbiome association with conditions

66 such as LTS in turkeys, it is therefore necessary to better understand the succession of bacterial

67 communities in the gastrointestinal tract as the bird ages prior to successfully modulating these

68 microbes using antibiotic-free approaches. Thus, the purpose of this study was to examine

69 bacterial community succession in turkeys raised under industry conditions, and to compare the

70 bacterial communities of young turkeys of different average flock weights using 16S rRNA

71 microbiome analysis.

\section{Materials and Methods}

Sample Collection. All animal experiments were performed in accordance with the

74 Institutional Animal Care and Use Committee at the University of Minnesota, protocol

75 1012B93592. Two independent experiments were performed. In experiment \#1, we examined

76 flock-level differences in the ileum bacterial microbiome using pooled samples from multiple

77 turkey flocks representing flocks of differing average daily weight. Five commercial flocks

78 (designated flocks \#1-2, \#4, and \#7-8) from three different facilities were examined, and two

79 research flocks (designated flocks \#5-6) were examined (Table 1). At three time points (1, 2, and

803 weeks of age, +/- 3 days), 20-40 birds were selected per flock/timepoint and humanely

81 euthanized using AVMA approved methods. Ileal sections of the small intestine were aseptically

82 collected, homogenized and immediately frozen. Homogenates included both intestinal content

83 and intestinal wall to assess the total bacterial content in the ileum. Sample poult weights for each

84 flock were determined at each collection timepoint. 
to 12 weeks of age (Table 1). From weeks 1-6 of age, birds were sampled at weekly timepoints,

87 followed by sampling every two weeks through 11 or 12 weeks of age. Individual birds were

88 euthanized and their ileum contents collected as described above. For each euthanized bird, total

89 bird weights, intestinal weights, and intestinal lengths were recorded.

pooled together according to flock, time point and weight status. For experiment \#2, individuality

of the samples was retained. DNA was extracted using a bead-beating procedure and the

94 The V3 hypervariable region of the 16S rRNA gene was amplified in 25ul reactions containing

95 1X PCR buffer (containing $1.8 \mathrm{mM} \mathrm{MgCl}_{2}$ ), $0.2 \mathrm{mM}$ each dNTP (Promega, Madison, WI),

Fidelity Taq polymerase (Roche, Basel, Switzerland). Primers were designed for Illumina

reverse primer contained a sample-specific sequence barcode. The PCR conditions used were an initial denaturation of $95^{\circ} \mathrm{C}$ for $2 \mathrm{~min}$, followed by 25 cycles of $95^{\circ} \mathrm{C}$ for $30 \mathrm{sec}, 60^{\circ} \mathrm{C}$ for $30 \mathrm{sec}$ and $72^{\circ} \mathrm{C}$ for $30 \mathrm{sec}$; the amplification was completed with a final extension of $72^{\circ} \mathrm{C}$ for $7 \mathrm{~min}$. The PCR product was excised from a 1.5\% gel and purified using the QIAquick Gel Extraction

103 Kit following manufacturer's instructions (Qiagen). Sample DNA quality and quantity were

104 assessed on a Bioanalyzer 2100 (Agilent, Palo Alto, CA) using a DNA-1000 lab chip. Sequencing

105 was performed at the University of Minnesota using Illumina MiSeq paired-end 2X250 bp 106 technology. 
109 (Masella et al. 2012). To reduce the effects of random sequencing errors, sequences that met any

110 of the following criteria were eliminated: sequences that did not match the PCR primers and

111 barcode; sequences that were truncated; sequences with one or more undetermined nucleotide

$112(\mathrm{~N})$; and sequences with an average Phred score $\leq 27$. Proximal and distal primers were trimmed

113 from the remaining sequence reads prior to database searches and similarity calculations. A de

114 novo operational taxonomic unit (OTU) picking approach was used in QIIME (Caporaso et al.

$115 \underline{2010})$ using uclust (Edgar 2010). Potential chimeras were removed using ChimeraSlayer (Edgar

116 2010). Approximately-maximum-likelihood phylogenetic trees were constructed using FastTree

117 (Price et al. 2010). QIIME was also used for assessments of alpha diversity, beta diversity using

118 Unifrac (Lozupone \& Knight 2005), and phylogenetic classifications using the RDP database

119 (Cole et al. 2009; Wang et al. 2007). Differential abundances of OTUs and other phylogenetic

120 classifications were identified using METASTATS (White et al. 2009). Construction of heatmaps

121 was performed using the R statistical software (Dean \& Nielsen 2007). MEGA5 was used for

122 inferring the phylogenetic relationships between known Lactobacillus species and similar OTUs

123 (Tamura et al. 2011). A cladogram depicting OTU relationships was developed using the

124 Interactive Tree of Life (iTOL) (Letunic \& Bork 2007).

Light Microscopy. From 20 individual samples with known variations in abundance of an

126 OTU similar to Candidatus division Arthromitus, $0.25 \mathrm{~g}$ of homogenized material was diluted in

$12710 \mathrm{ml}$ phosphate buffered saline. From this sample, $10 \mathrm{ul}$ was fixed onto a microscope slide and

128 stained with crystal violet. Five fields per sample were used to count and average segmented

129 filamentous bacteria within the field.

130 Results

131 A total of 140 samples were analyzed in this study, involving pooled samples for flock-

132 level comparisons (experiment \#1) and individual samples for within-flock comparisons 
133 (experiment \#2). From these samples, a total of 18,117,871 2X250 bp Illumina MiSeq reads were

134 generated, assembled and quality screened using Pandaseq, trimmed of primer/barcode, and 135 rarefied to 2,776,725 sequences. These remaining sequences were analyzed using QIIME (Table

136 1). After removal of singleton OTUs upon clustering, there were 2,469 OTUs that remained in the 137 dataset representing species-level OTUs across all samples at 97\% clustering.

138 Experiment \#1: flock-level comparisons.

139

According to average daily poult weights, flocks were classified into two groups, light

140 (flocks \#1-4 and \#7) and heavy (flocks \#5-6 and \#8) which were significantly different from one

141 another based upon weight $(\mathrm{P}<0.05$; Fig. 1). Subsequent comparisons of sequencing data were

142 based upon these classifications. In total, 396,725 16S rRNA sequences were analyzed for flock-

143 level comparisons (Table 1).

144 RDP was used to analyze 16S rRNA sequence reads at the class level, using a bootstrap 145 confidence threshold of 50\% based upon RDP recommendations for short read lengths (Cole et

146 al. 2009). Using RDP analysis, Firmicutes was the most prevalent phylum in all flocks for the

147 duration of the experiment (Fig. 2), comprised mostly of Bacilli. Gammaproteobacteria made up

148 substantial proportions of the bacterial microbiome at week 1 of age in both the Heavy and Light

149 flocks. Genus-level distribution showed that these populations were largely composed of

150 Pseudomonas species in flocks \#1 and \#5, but were mostly Escherichia coli in flock \#8. Flocks

$151 \# 1$ and \#2 also showed a higher occurrence of the class Actinobacteria than the other five flocks,

152 which was composed mostly of the genus Bifidobacterium. Overall, the RDP analysis showed on

153 the class level that Bacilli and Actinobacteria were significantly higher $(\mathrm{P}<0.05)$ on average in

154 the Light flocks. The Heavy flocks had higher proportions of an unknown bacteria at weeks 2 and

1553 of age, which was later identified as Candidatus division Arthromitus (see below). 
157 discernible pattern in any of the 7 flocks (Table S1). This is also demonstrated through Chao1,

158 Shannon and Simpson richness and diversity indices, again suggesting an immature and changing

159 gut microbiome during the first three weeks of life. The rarefaction curve (Fig. S1) also suggested

160 that there was no discernable pattern to the curves from weeks 1 to 3 of age in the flocks; if the

161 diversity and richness was increasing over the 21 day time period of this study, one might expect

162 the slope of the curves from weeks 1 to 3 of age to also increase accordingly. Inferred phylogeny

163 of the collective turkey ileum microbiome based upon OTU (Fig. 3) was comparable to the

164 taxonomic classifications performed with RDP, with most of the diversity of the microbiome

165 being attributed to orders Clostridia and Bacilli. Unifrac-based distance matrices demonstrated

166 that there were no significant differences in the overall microbiome compositions of Light versus

167 Heavy flock at any of the three timepoints (Fig. S2). However, OTU-based analysis revealed 168 significant changes in bacterial taxa, as described below.

169 Among the observed OTUs in the entire dataset, the ten most abundant OTUs represented

$17078.6 \%$ of the total sequences obtained (Table S2). These dominant OTUs were classified using

171 RDP database and BlastN, and included representative sequences with similarity to Lactobacillus

172 salivarius, Lactobacillus acidophilus/crispatus/helveticus (L. delbrueckii group), Lactobacillus

173 aviarius, Lactobacillus johnsonii, Clostridium bartlettii (Clostridium group XI), Lactobacillus

174 reuteri/vaginalis, Candidatus division Arthromitus, Enterococcus species, E. coli, and Roseburia

175 species. In some cases, the V3 region was sufficient for BLAST similarity enabling classification

176 down to the species level at $100 \%$ similarity to database sequences, as demonstrated for certain

177 Lactobacillus species in Fig. 4. The dominant OTUs were not evenly distributed among the

178 flocks examined. In fact, a clear trend was observed where the flocks classified as Heavy

179 contained greater proportions of OTUs classified as Candidatus division Arthromitus and $C$. 
bartlettii, and lesser proportions of OTUs classified as Lactobacillus species. METASTATS was

181 performed to determine significant enrichments or depletions in OTUs in the flocks classified as

182 Light" versus those classified as Heavy (Table S3). We chose METASTATS because it employs a

183 false discovery rate to deal with high-complexity environments and uses Fisher's exact test to

184 deal with low abundance or sparsely sampled features (White et al. 2009). At week 1 of age,

185 significantly higher proportions $(\mathrm{P}<0.05)$ of OTUs were observed in Heavy flocks with similarity

186 to Anaerobacter species, Clostridium sensu stricto, and Pseudomonas species. In birds two weeks

187 of age, most of the significant changes in Light versus Heavy flocks included higher proportions

188 in OTUs classified as Clostridium bartlettii and Candidatus division Arthromitus and depletions

189 in a number OTUs classified as Lactobacillus spp. in Heavy flocks. At birds three weeks of age,

190 reduced proportions were also observed in OTUs classified as Lactobacillus species along with

191 increases in the proportions of Candidatus division Arthromitus OTU and several OTUs

192 classified as Lactococcus species in Heavy flocks. Light microscopy was used to validate that

193 sequences with similarity to Candidatus division Arthromitus appeared as segmented filamentous

194 bacteria (Fig. 5). Indeed, segmented filamentous organisms were easily identified in samples that

195 contained large proportions of sequences with similarity to Candidatus division Arthromitus,

196 while these organisms were absent in samples lacking sequences with similarity to Candidatus

197 division Arthromitus. The average number of organisms identified in 5 fields per sample

198 correlated with the counts of the OTU similar to Candidatus division Arthromitus $\left(\mathrm{R}^{2}=0.92\right)$.

199 Experiment \#2: within-flock comparisons.

200 In experiment \#2, two flocks were followed from ages 1-12 weeks and individual ileum

201 bacterial microbiomes were compared. A total of 119 individual turkey ileum samples were

202 collected from multiple birds at each timepoint examined, resulting in 2,380,000 sequences that

203 were analyzed in QIIME. The dominant OTUs identified in experiment \#1 were the same 
204 dominant OTUs identified in experiment \#1. However, following individual birds over extended

205 periods of time enabled better resolution related to the succession of bacterial populations in the 206 turkey ileum. The flocks followed included a typical commercial turkey flock (designated CF) of 20728,000 hens and a research flock (designated RF) replicating commercial farm conditions. Both

208 flocks were sampled at approximately the same timeframe, used the same hatchery as a source of 209 poults, and used similar nutritional plans. The two flocks were strikingly similar in their ileum 210 bacterial microbiome succession, with no significant differences at community-level comparisons 211 based on average Unifrac distances at the same age timepoints (Fig. S3). An average heatmap 212 was constructed to visualize OTU averages for each flock at each timepoint (Fig. 6). In both 213 flocks, birds possessed high proportions of OTUs with similarity to L. salivarius, $L$.

214 reuteri/vaginalis, and L. acidophilus/crispatus/helveticus (L. delbrueckii group) throughout the 12 215 weeks of the study. In contrast, OTUs with similarity to L. aviarius and L. johnsonii appeared at 216 2-4 weeks of age and subsequently increased or maintained their proportions with age in both 217 flocks. An OTU with similarity to C. bartlettii appeared in both flocks at week 5 of age. A 218 number of lower abundance OTUs also appeared over time in a predictable manner in both flocks 219 studied. One key difference between the two flocks studied was the timing of the appearance of 220 the OTU with similarity to Candidatus division Arthromitus, increasing in abundance at 4 weeks 221 of age in the commercial flock and at 2 weeks of age in the research flock. While the proportions 222 of some OTUs varied in individual birds of the same flock and timepoint, overall there were 223 discernable patterns and consistency in the temporal succession of the bacterial microbiome in 224 individual birds (Fig. 7). Sample diversity was assessed temporally using the Shannon index as a 225 measure of community diversity and Chao1 as an estimator of community richness (Fig. S4), and 226 this demonstrated that diversity and richness increased temporally until 8 weeks of age where it 227 plateaued. 
229 bird age was more influential than flock or Heavy/Light classification (Fig. 8). Also evident was

230 that as turkey age increased, the individual birds shared greater bacterial community similarity,

231 particularly after the move of birds from brood to grow-out. PCoA coordinate PC2 was plotted

232 against individual bird whole bird weights, and demonstrated that there was a predictable shift in

233 the bacterial community from hatch through approximately $3 \mathrm{~kg}$ of weight, after which the ileum

234 microbiome stabilized (Fig. S5). Whole bird weight also was highly correlated with intestinal

235 weight $\left(\mathrm{R}^{2}=0.83\right.$; Fig. S6). Based upon two-way hierarchical clustering using the top 50 OTUs

236 in the study, the Neighbor Joining cladogram illustrated that the Heavy flocks in experiment \#1

237 clustered independently from Light flocks (Fig. 9). Overall, the clustering enabled not only

238 clustering based upon bird age, but also clustering based upon flock source.

The core microbiome was also assessed using the data from flocks CF and RF over all timepoints (Table S4). The core microbiome was defined as OTUs present in $100 \%$ of all samples

241 at a given timepoint. From these data, a proposed model for the succession of the ileum

242 microbiome was constructed (Fig. 10) involving the dominant bacteria that are considered core at 243 different timepoints as the turkey ages.

\section{Discussion}

The purpose of this study was to identify differences in the bacterial microbiome in

246 commercial turkeys of differing average flock weights, and the succession of the ileum bacterial

247 microbiome in the growing commercial turkey. We hypothesized that differences in bacterial

248 microbiome content could be identified that correlated with Heavy versus Light groups based

249 upon average flock weights. In previous work, we demonstrated that age was the major driving 
250 factor in the chicken cecum bacterial microbiome when examining a single research flock, as

251 compared to lesser community effects of in-feed antibiotic treatments (Danzeisen et al. 2011).

252 Age was also a dominating factor in the bacterial microbiomes in this study, but environment also

253 appears to play a key role in the initial stages of turkey bacterial microbiome maturation. Using

254 RDP-based taxonomic classification, discernable patterns between Light and Heavy flocks were

255 identified that included decreases in Bacilli and increases in unclassified bacteria later identified

256 as Candidatus division Arthromitus. OTU-based analysis at 97\% similarity enabled much greater

257 resolution of the finer-scale microbiome changes occurring between Light and Heavy flocks, and

258 also microbiome changes as the turkey ages. Our data suggest that certain bacterial taxa can be

259 identified that may be important in the early development of the turkey small intestine.

260 Some of the predominant OTUs identified in this study (with similarity to Candidatus

261 division Arthromitus, L. aviarius, and L. salivarius) have also been identified as predominant

262 species in the chicken mucosal microbiota (Gong et al. 2007). While Lactobacillus species are

263 commonly used in commercial agricultural probiotics and are considered to be positively

264 correlated with gut health, we observed an inverse correlation between bird performance and

265 Lactobacillus species abundance when comparing Light versus Heavy flocks. There is

266 precedence for this in a previous study involving broiler chickens, where it was found that $L$.

267 salivarius and L. aviarius were associated with decreased bird performance (Torok et al. 2011).

268 These and other studies therefore suggest that an excess abundance of certain Lactobacillus

269 species in the avian small intestine may actually be indicative of decreased or slower bird

270 development, and this could in fact be due to displacement with other bacteria such as Candidatus

271 division Arthromitus and Clostridium group XI organisms that are critical for gut microbiome

272 development (Danzeisen et al. 2011). 
274 developing microbiota in the animal small intestine. These bacteria are visible in the ileum of

275 turkey poults in the early stages of life (Bohorquez et al. 2011). Previously, SFBs have mostly

276 been associated with disease in poultry (Angel et al. 1990; Goodwin et al. 1991). However, these

277 organisms are quite heterogeneous and belong to multiple bacterial taxa (Thompson et al. 2013).

278 Therefore, SFBs from different bacterial taxa may exert different effects on the poultry

279 gastrointestinal tract. Some SFBs belong to the candidate taxa known as Candidatus division

280 Arthromitus, more recently referred to as Candidatus Savagella ( $\underline{\text { Snel et al. 1995; Thompson et al. }}$

281 2013). Phylogenetically speaking, these organisms form a discrete and distant lineage within the

282 family Clostridiaceae, clustering within Clostridia Cluster I but divergent from other organisms

283 within this cluster (Prakash et al. 2011). Candidatus division Arthromitus organisms are Gram-

284 positive, spore-forming bacteria that are of long filamentous form consisting of segmented

285 structures. Candidatus division Arthromitus organisms are unique from other Clostridia in that

286 they have reduced genomes and are thus highly dependent on their host for many metabolic

287 functions, including synthesis of amino acids and purines and pyrimidines. Because of these

288 dependencies, they have not yet been cultured. Interestingly, Candidatus division Arthromitus

289 organisms have been previously associated with early development of the innate immune system

290 in mice and have been positively associated with gut development in other animal species

291 (Prakash et al. 2011). Therefore, whether directly or indirectly, our data suggest that Candidatus

292 division Arthromitus-like organisms play a positive role in turkey gut development and weight

293 gain.

While we found a clear correlation between the presence of Candidatus division

295 Arthromitus-like organisms and Heavy flock status, it is important to note that the sequence

296 abundance of this OTU was among the most variable identified based upon standard deviations. 
297 That is, in individual birds the presence or absence of this organism was highly unpredictable,

298 although the average abundance of this organism using a pooled timepoint approach showed clear

299 trends. This may underscore the reality that substantial inconsistencies exist between poults

300 entering the brood environment that may be contributing to their variable temporal microbiome

301 succession. Future studies are essential that address not only the bacterial microbiome in the

302 turkey gastrointestinal tract, but also the bird immune status that corresponds with these

303 microbiota changes.

304

In addition to Candidatus division Arthromitus, several other OTUs were identified that

305 were associated with Heavy flocks and/or associated with temporal shifts in the turkey ileum

306 microbiome. L. aviarius is a Gram-positive, non-spore-forming strict anaerobe that was first

307 isolated and identified from the chicken (Fujisawa 1984). L. aviarius was found to be suppressed

308 upon Clostridium perfringens challenge in broiler chickens (Feng et al. 2010), and was also

309 suppressed following salinomycin treatment in broilers (Czerwinski et al. 2012). Therefore, the

310 emergence of L. avaiarius in the small intestine appears to be significant in terms of bird gut

311 microbiota development and warrants further study.

Another OTU that was a clear marker of ileum microbiome succession in this study was

$313100 \%$ similar to C. bartlettii. This bacteria belongs to group XI Clostridium (Song et al. 2004),

314 which is a heterogeneous group of Clostridium that also includes Eubacterium and

315 Peptostreptococcus species. C. bartlettii is poorly described in the literature, but it has been

316 associated with a high ability to ferment aromatic amino acids in the gut (Russell et al. 2013).

317 Since L. aviarius and C. bartlettii are strict anaerobes, it is also possible that the gastrointestinal

318 environment dictates the ability of these organisms to colonize and expand in numbers. Whatever

319 the case, these bacteria appear to be good markers of ileum bacterial microbiome development in 320 the turkey. 
322 there was a clear shift in the microbiome that occurred in all flocks examined. The timing of this

323 shift varied between flocks examined, and in general the shift occurred earlier in research flocks

324 than it did in commercial flocks. Clustering analysis indicated that the largest differences in the

325 timing of this shift occurred in the Heavy research flocks in experiment \#1. This is easily

326 explained by the nature of these research flocks, which were pen-based studies, compared to all

327 other flocks that were either actual commercial flocks or research flocks representing commercial

328 conditions. While the timing of bacterial microbiome succession varied depending on flock

329 studied, the succession of microbes was clear (Fig. 10) and a predictable core microbiome was

330 established that was present in $100 \%$ of the birds examined at each timepoint (Table S4). Our

331 model for the succession of bacteria in the turkey ileum, based upon this study, indicates that the

332 core predictable bacterial microbiome in the turkey involves early colonization with

333 Gammaproteobacteria at high variability (particularly E. coli). At the same time, consistent and

334 dominant colonization occurs with L. delbrueckii group organisms, L. salivarius, and L.

335 reuteri/vaginalis, along with colonization of lower abundance organisms including Blautia and

336 Roseburia species. A shift occurs at weeks 3-4 of age when some birds are colonized heavily but

337 variably by Candidatus division Arthromitus, which is accompanied by consistent colonization by

338 L. aviarius and L. johnsonii, along with colonization of lower abundance organisms including

339 Bifidobacterium and Corynebacterium species. At weeks 5-7 of age, Clostridium group XI

340 organisms and L. ingluviei begin to consistently colonize the ileum. The overall timing of the key

341 shifts appears to occur earlier in research flocks as compared to commercial flocks. The reasons

342 for this observation are currently unknown. We can at least rule out poult source and diet, since

343 the birds in this study came from the same hatchery and were fed similar diets. Other possible

344 mitigating factors include management approaches and overall bird immune status, and these are

345 variables requiring further examination. 
347 timepoint in experiment \#1, limiting our ability to assess individual-to-individual variation within

348 a given flock when comparing Light versus Heavy flocks. This was better addressed in

349 experiment \#2 addressing individual-to-individual turkey variation. Another limitation is that we

350 performed sequencing on a small portion of the 16S rRNA gene, so the data obtained here are not

351 indicative of functional aspects of the turkey small intestinal microbiota. Furthermore, we have a

352 high level of confidence that the Candidatus Arthromitus-like organisms are SFBs associated

353 with the ileum mucosa through DNA sequencing and light microscopy, but additional tools such

354 as in situ hybridization would be required to confirm these observations. Furthermore, this work

355 did not address viable counts of identified bacteria so it only measured the presence or absence of 356 DNA representing these bacteria.

\section{Conclusions}

This study demonstrates that the succession of bacteria in the turkey ileum proceeds in a

359 predictable manner. The timing of bacterial succession appears to be dependent on multiple

360 factors, at least including incoming poult consistency and commercial flock environment. From

361 this work, several candidate markers of turkey bacterial microbiome development have been

362 identified that will aid in future efforts aimed at modulating the microbiome to improve turkey

363 gut health and overall bird growth and development.

\section{Acknowledgements}


366 graciously participated in this study. Bioinformatics was enabled through support from the

367 Minnesota Supercomputing Institute. We thank Dr. Richard Isaacson and Dr. Srinand Sreevatsan 368 at the University of Minnesota for thoughtful and enlightening discussions. 


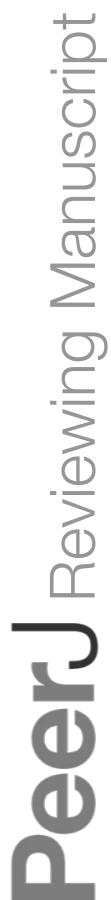

PeerJ reviewing PDF | (v2013:04:418:2:0:NEW 10 Dec 2013) 
370

371

372

373 374

375

376

377

378

379

380

381

382

383

384

385

386

387

388

389

390

391

392

Angel CR, Sell JL, Fagerland JA, Reynolds DL, and Trampel DW. 1990. Long-segmented filamentous organisms observed in poults experimentally infected with stunting syndrome agent. Avian Dis 34:994-1001.

Bartram AK, Lynch MD, Stearns JC, Moreno-Hagelsieb G, and Neufeld JD. 2011. Generation of multimillion-sequence $16 \mathrm{~S}$ rRNA gene libraries from complex microbial communities by assembling paired-end illumina reads. Appl Environ Microbiol 77:3846-3852.

Bohorquez DV, Bohorquez NE, and Ferket PR. 2011. Ultrastructural development of the small intestinal mucosa in the embryo and turkey poult: A light and electron microscopy study. Poult Sci 90:842-855.

Calvert A. 2012. Light turkey syndrome: field study and inoculation trial. Master Thesis. Department of Animal Sciences, University of Minnesota.

Caporaso JG, Kuczynski J, Stombaugh J, Bittinger K, Bushman FD, Costello EK, Fierer N, Pena AG, Goodrich JK, Gordon JI et al. . 2010. QIIME allows analysis of high-throughput community sequencing data. Nat Methods 7:335-336.

Cole JR, Wang Q, Cardenas E, Fish J, Chai B, Farris RJ, Kulam-Syed-Mohideen AS, McGarrell DM, Marsh T, Garrity GM et al. . 2009. The Ribosomal Database Project: improved alignments and new tools for rRNA analysis. Nucleic Acids Res 37:D141-145.

Czerwinski J, Hojberg O, Smulikowska S, Engberg RM, and Mieczkowska A. 2012. Effects of sodium butyrate and salinomycin upon intestinal microbiota, mucosal morphology and performance of broiler chickens. Arch Anim Nutr 66:102-116.

Danzeisen JL, Kim HB, Isaacson RE, Tu ZJ, and Johnson TJ. 2011. Modulations of the chicken cecal microbiome and metagenome in response to anticoccidial and growth promoter treatment. PLoS One 6:e27949. 
393 Dean CB, and Nielsen JD. 2007. Generalized linear mixed models: a review and some extensions. Lifetime Data Anal 13:497-512.

395 Edgar RC. 2010. Search and clustering orders of magnitude faster than BLAST. Bioinformatics

396 26:2460-2461.

397 Feng Y, Gong J, Yu H, Jin Y, Zhu J, and Han Y. 2010. Identification of changes in the 398 composition of ileal bacterial microbiota of broiler chickens infected with Clostridium perfringens. Vet Microbiol 140:116-121.

400 Fujisawa T, Shirasaka, S., Watabe, J., Mitsuoka, T. 1984. Lactobacillus aviarius sp. nov.: A new 401 species isolated from the intestine of chickens. Syst Appl Microbiol 5:414-420.

402 Gong J, Si W, Forster RJ, Huang R, Yu H, Yin Y, Yang C, and Han Y. 2007. 16S rRNA gene403 based analysis of mucosa-associated bacterial community and phylogeny in the chicken 404 gastrointestinal tracts: from crops to ceca. FEMS Microbiol Ecol 59:147-157.

405 Goodwin MA, Cooper GL, Brown J, Bickford AA, Waltman WD, and Dickson TG. 1991.

406

407

408 Clinical, pathological, and epizootiological features of long-segmented filamentous organisms (bacteria, LSFOs) in the small intestines of chickens, turkeys, and quails. Avian

409 Handelsman J. 2004. Metagenomics: application of genomics to uncultured microorganisms. Microbiol Mol Biol Rev 68:669-685.

411 Jindal N, Chander Y, Patnayak DP, Mor SK, Ziegler AF, and Goyal SM. 2012. A multiplex RT412 PCR for the detection of astrovirus, rotavirus, and reovirus in turkeys. Avian Dis 56:592596.

414 Jindal N, Patnayak DP, Chander Y, Ziegler AF, and Goyal SM. 2010. Detection and molecular 415 characterization of enteric viruses from poult enteritis syndrome in turkeys. Poult Sci

416 $89: 217-226$. 
417 Jindal N, Patnayak DP, Ziegler AF, Lago A, and Goyal SM. 2009. Duration of growth depression 418 and pathogen shedding in experimentally reproduced poult enteritis syndrome. Avian Dis 53:517-522.

420 Kim GB, Seo YM, Kim CH, and Paik IK. 2011. Effect of dietary prebiotic supplementation on 421 the performance, intestinal microflora, and immune response of broilers. Poult Sci 90:7582.

Letunic I, and Bork P. 2007. Interactive Tree Of Life (iTOL): an online tool for phylogenetic tree 424 display and annotation. Bioinformatics 23:127-128.

Lozupone C, and Knight R. 2005. UniFrac: a new phylogenetic method for comparing microbial communities. Appl Environ Microbiol 71:8228-8235.

Masella AP, Bartram AK, Truszkowski JM, Brown DG, and Neufeld JD. 2012. PANDAseq: paired-end assembler for illumina sequences. BMC Bioinformatics 13:31.

Mor SK, Abin M, Costa G, Durrani A, Jindal N, Goyal SM, and Patnayak DP. 2011. The role of type-2 turkey astrovirus in poult enteritis syndrome. Poult Sci 90:2747-2752.

Mor SK, Sharafeldin TA, Abin M, Kromm M, Porter RE, Goyal SM, and Patnayak DP. 2013. The occurrence of enteric viruses in Light Turkey Syndrome. Avian Pathol 42:497-501.

433 Prakash T, Oshima K, Morita H, Fukuda S, Imaoka A, Kumar N, Sharma VK, Kim SW, Takahashi M, Saitou N et al. . 2011. Complete genome sequences of rat and mouse segmented filamentous bacteria, a potent inducer of Th17 cell differentiation. Cell Host Microbe 10:273-284.

437 Price MN, Dehal PS, and Arkin AP. 2010. FastTree 2--approximately maximum-likelihood trees $438 \quad$ for large alignments. PLoS One 5:e9490.

439 Russell WR, Duncan SH, Scobbie L, Duncan G, Cantlay L, Calder AG, Anderson SE, and Flint 440 HJ. 2013. Major phenylpropanoid-derived metabolites in the human gut can arise from 441 microbial fermentation of protein. Mol Nutr Food Res 57:523-535. 
442 Scalzo S, Corkill JE, Shanks DJ, Rowan TG, Delaval J, Fleetwood A, Murphy M, and Hart CA.

443

444

445

446

447

448

449

450

451

452

453

454

455

456

457

458

459

460

461 Torok VA, Hughes RJ, Mikkelsen LL, Perez-Maldonado R, Balding K, MacAlpine R, Percy NJ, 462

463

464 2004. Phenotypic and genotypic changes in Salmonella enterica subsp. enterica serotype typhimurium during passage in intestines of broiler chickens fed on diets that included ionophore anticoccidial supplements. J Clin Microbiol 42:3399-3405.

Scupham AJ. 2009. Campylobacter colonization of the turkey intestine in the context of microbial community development. Appl Environ Microbiol 75:3564-3571.

Scupham AJ, Patton TG, Bent E, and Bayles DO. 2008. Comparison of the cecal microbiota of domestic and wild turkeys. Microb Ecol 56:322-331.

Snel J, Heinen PP, Blok HJ, Carman RJ, Duncan AJ, Allen PC, and Collins MD. 1995. Comparison of 16S rRNA sequences of segmented filamentous bacteria isolated from mice, rats, and chickens and proposal of "Candidatus Arthromitus". Int J Syst Bacteriol 45:780-782.

Song YL, Liu CX, McTeague M, Summanen P, and Finegold SM. 2004. Clostridium bartlettii sp. nov., isolated from human faeces. Anaerobe 10:179-184.

Tamura K, Peterson D, Peterson N, Stecher G, Nei M, and Kumar S. 2011. MEGA5: molecular evolutionary genetics analysis using maximum likelihood, evolutionary distance, and maximum parsimony methods. Mol Biol Evol 28:2731-2739.

Thompson CL, Mikaelyan A, and Brune A. 2013. Immune-modulating gut symbionts are not "Candidatus Arthromitus". Mucosal Immunol 6:200-201. and Ophel-Keller K. 2011. Identification and characterization of potential performancerelated gut microbiotas in broiler chickens across various feeding trials. Appl Environ Microbiol 77:5868-5878. 
465 USDA. 2007. Overview of the U. S. Turkey Industry. National Agricultural Statistics Service, 466 online resoure, http://usda.mannlib.cornell.edu/MannUsda/viewDocumentInfo.do?

$467 \quad$ documentID=1762.

468 USDA. 2012. Turkeys Raised. National Agricultural Statistics Service, online resoure, 469 http://usda.mannlib.cornell.edu/MannUsda/viewDocumentInfo.do?documentID=1498.

470 Wang Q, Garrity GM, Tiedje JM, and Cole JR. 2007. Naive Bayesian classifier for rapid 471 assignment of rRNA sequences into the new bacterial taxonomy. Appl Environ Microbiol $472 \quad 73: 5261-5267$.

473 White JR, Nagarajan N, and Pop M. 2009. Statistical methods for detecting differentially 474 abundant features in clinical metagenomic samples. PLoS Comput Biol 5:e1000352. 


\section{Figure 1}

Average flock weights for Light versus Heavy flocks in experiment \#1.

- To calculate the average sample flock weight, 40 birds from each flock were weighed or an average weight from the barn scale readings was used. Dashed line indicates genetic potential of bird type used.

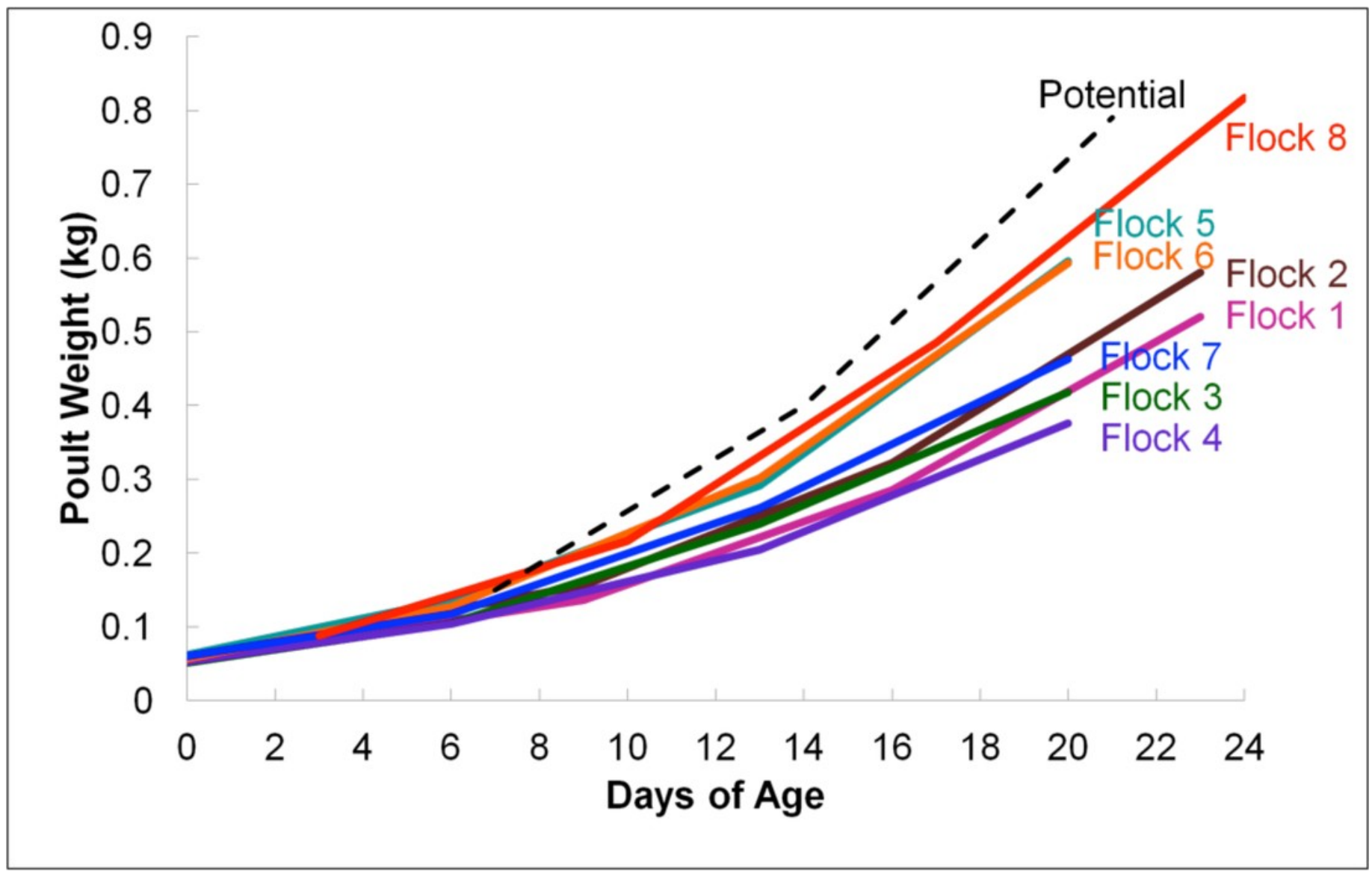




\section{Figure 2}

Taxonomic classification of groups in this study.

Class-level taxonomic classification was based upon average proportional abundance of normalized samples. Designations in the X-axis are "CF" = individual birds from commercial flock (experiment \#2), "RF" = individual birds from research flock (experiment \#2), "Heavy" = pooled samples categorized as heavy weights (experiment \#1), and "Light" = pooled samples categorized as lighter weights (experiment \#1). "W" = age of birds in weeks.

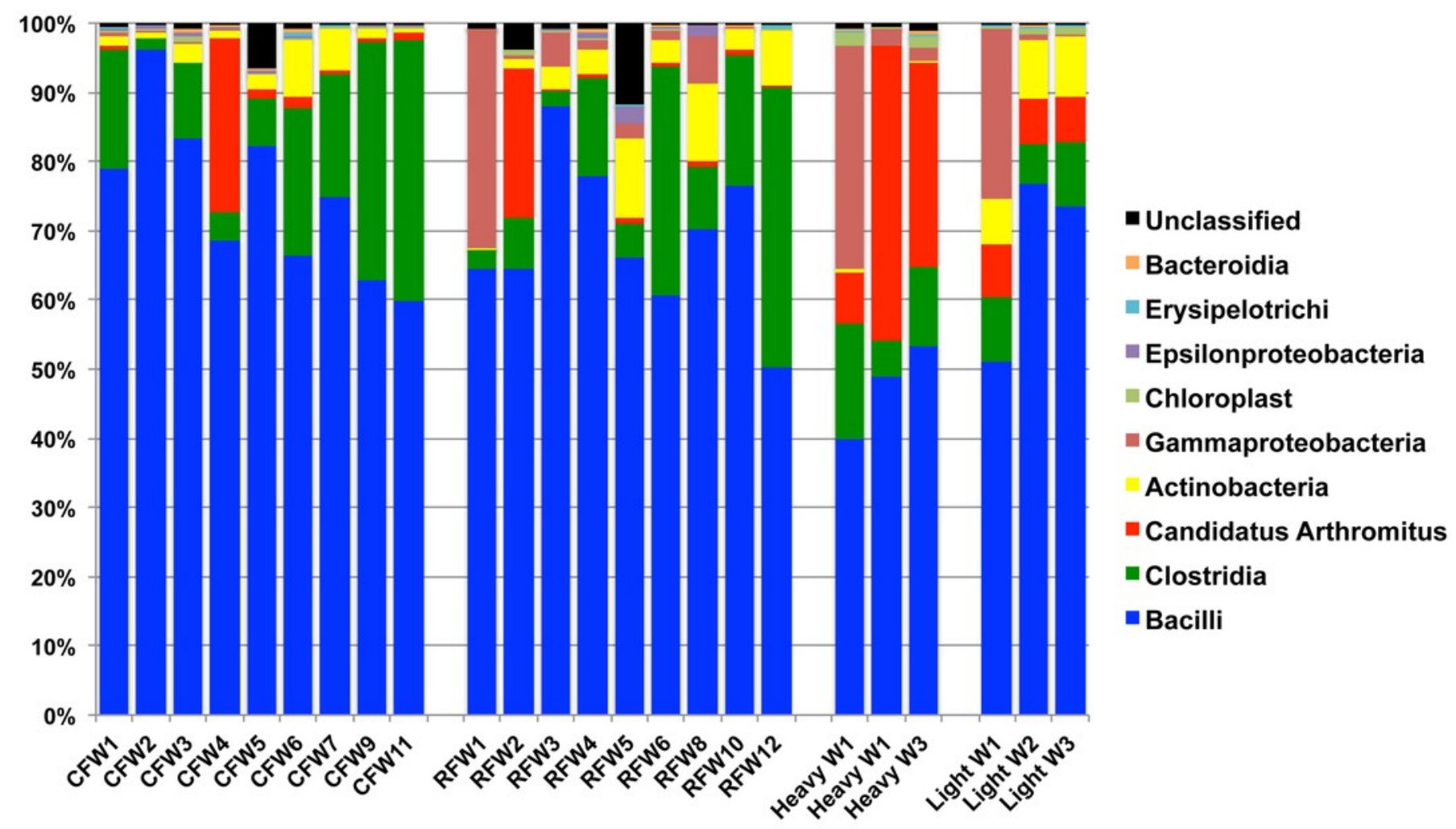




\section{Figure 3}

Cladogram of operational taxonomic units (OTUs).

Cladogram illustrating relationships of OTUs in the entire study, classified using the Ribosomal Database Project and/or BLAST. The outer ring depicts relative $\log _{10}$ abundances of OTUs in the entire dataset. Figure was generated using the Interactive Tree of Life (iTOL).

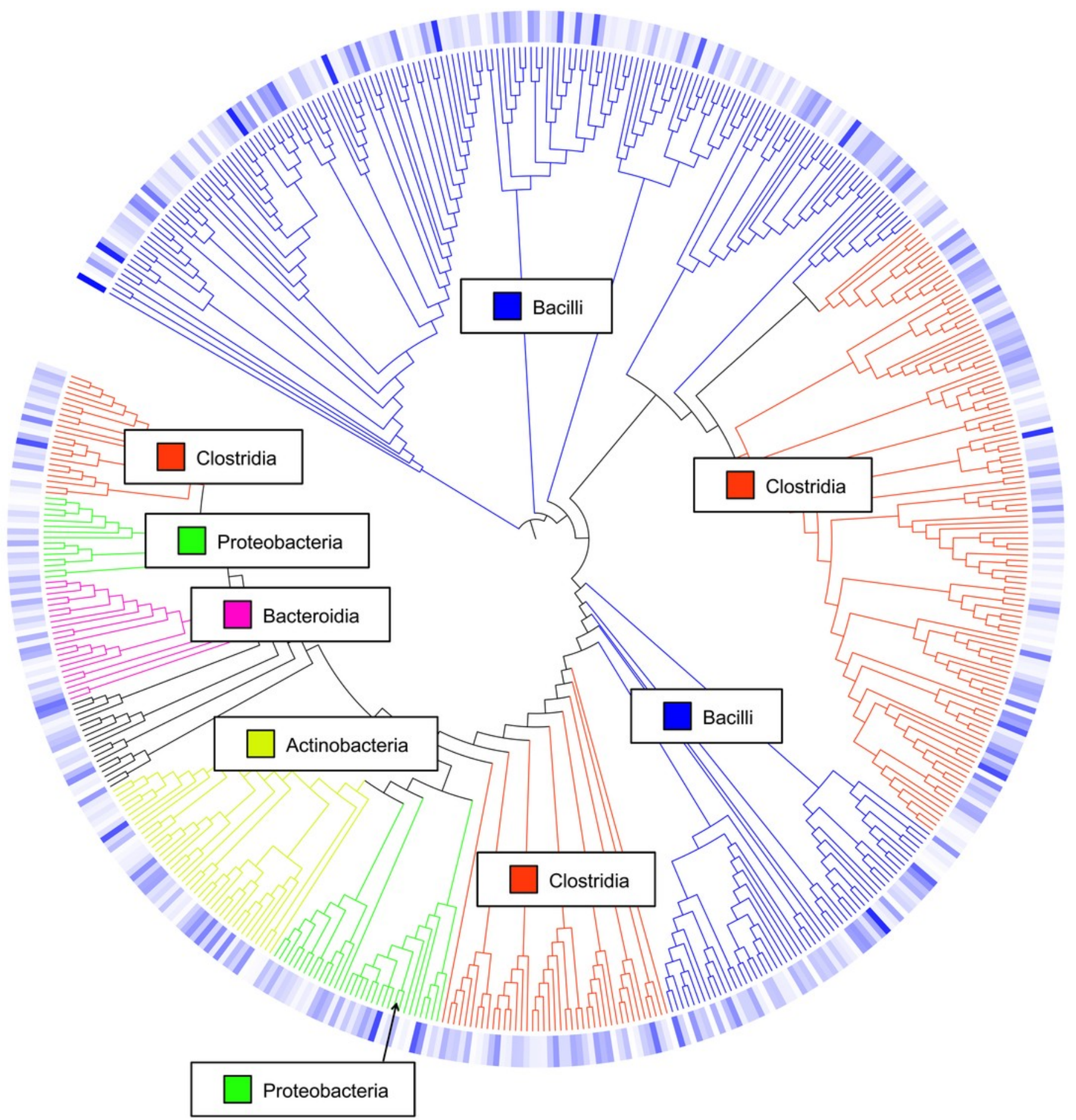




\section{Figure 4}

Dendrogram of operational taxonomic units (OTUs) with similarity to Lactobacillus.

Phylogenetic relationships were inferred using Maximum Likelihood analysis with a General Time Reversible Model using 1,000 bootstrap replicates in MEGA5. The dataset was generated from representative OTUs with similarity to Lactobacillus spp., and extracted Lactobacillus sequences from the NCBI database for the V3 hypervariable region of the $16 \mathrm{~S}$ rRNA region. 


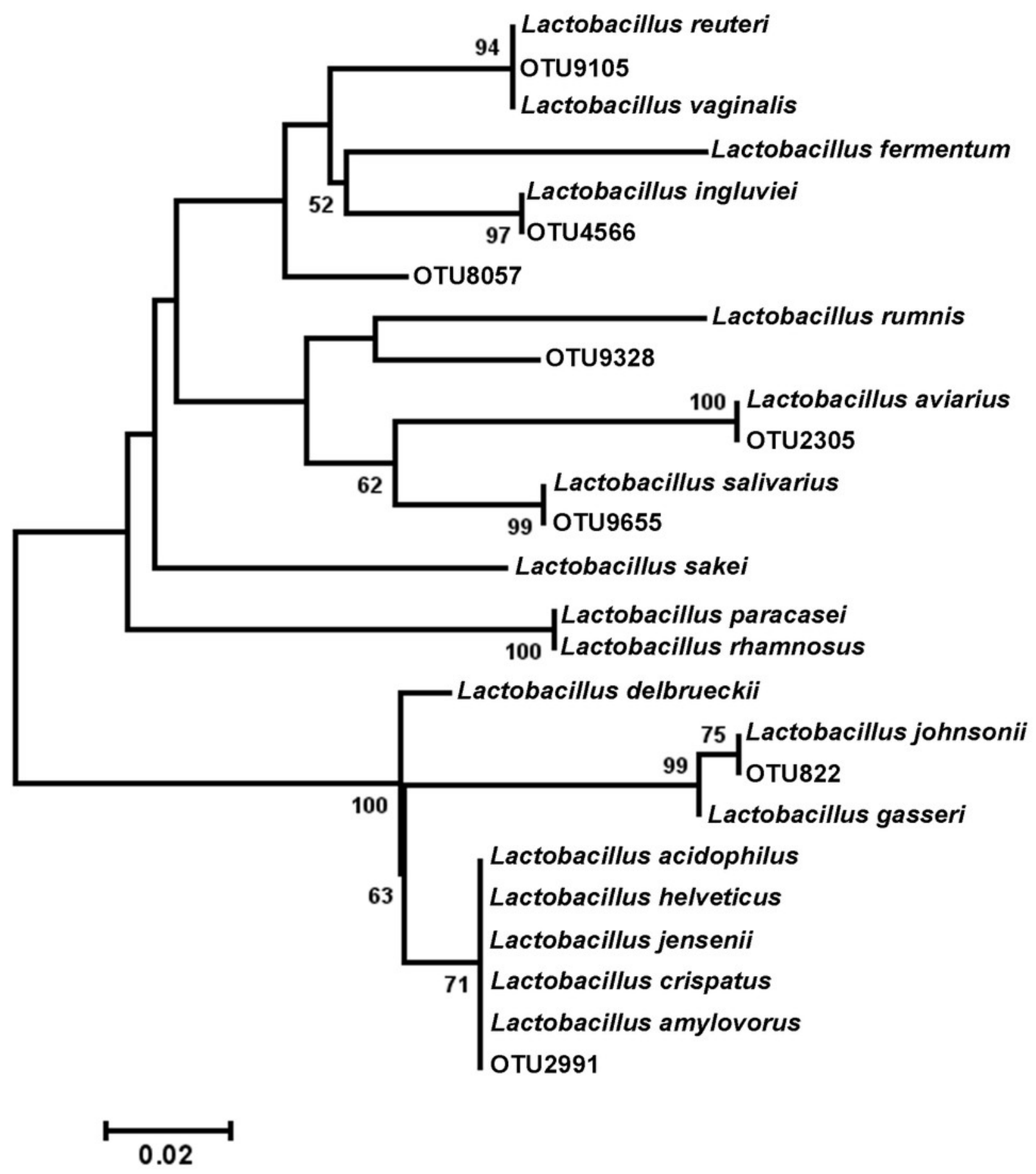




\section{Figure 5}

Light microscopy of segmented filamentous bacteria in the turkey ileum.

Scaled line is approximately 10 microns. 


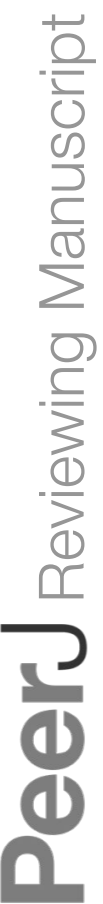

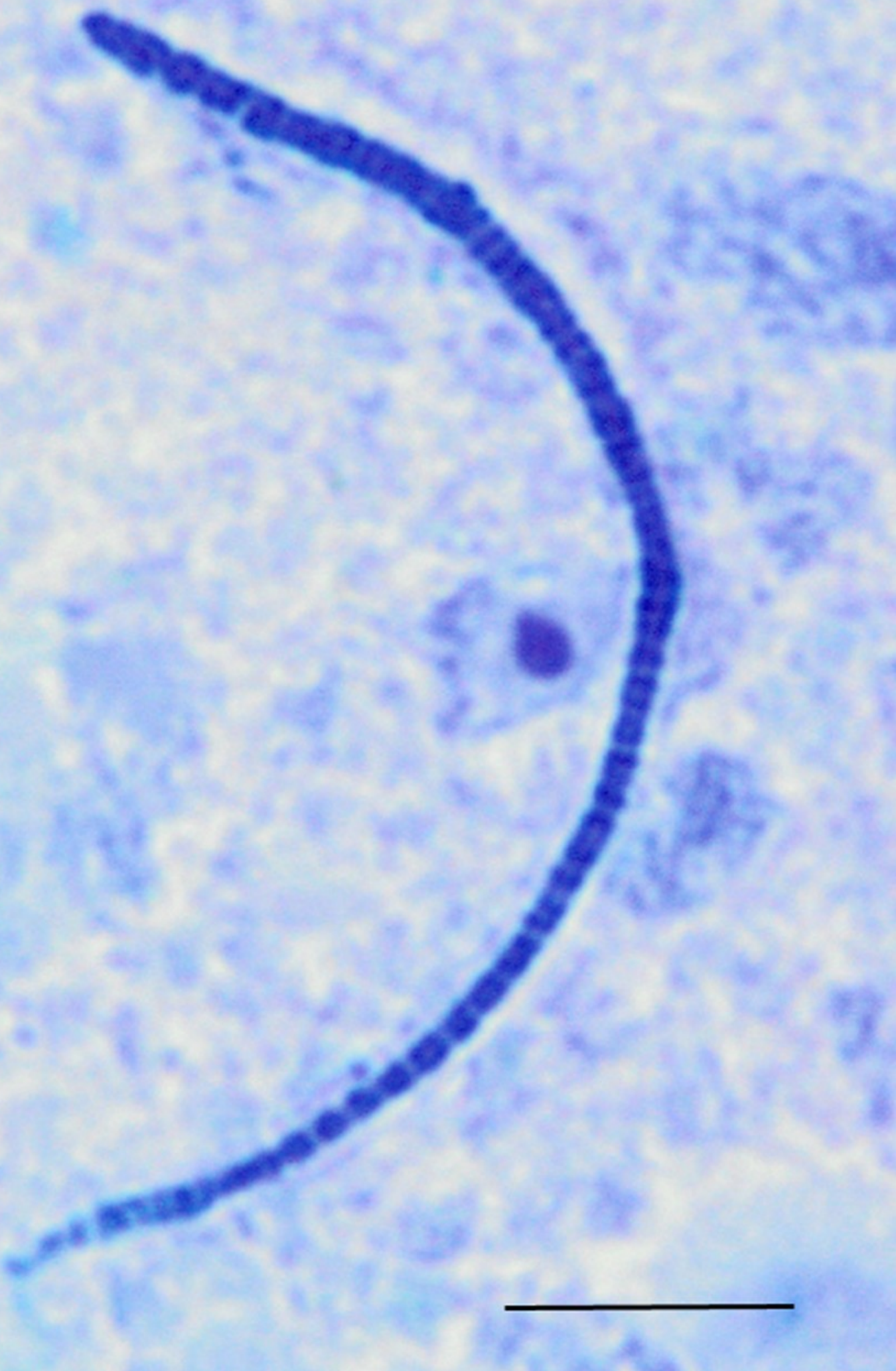




\section{Figure 6}

Heatmap of average OTU abundance for each group studied.

Averages for multiple birds at each group and timepoint are depicted for the top 50 OTUs in this study. Heatmap is in $\log _{10}$ normalized counts. 

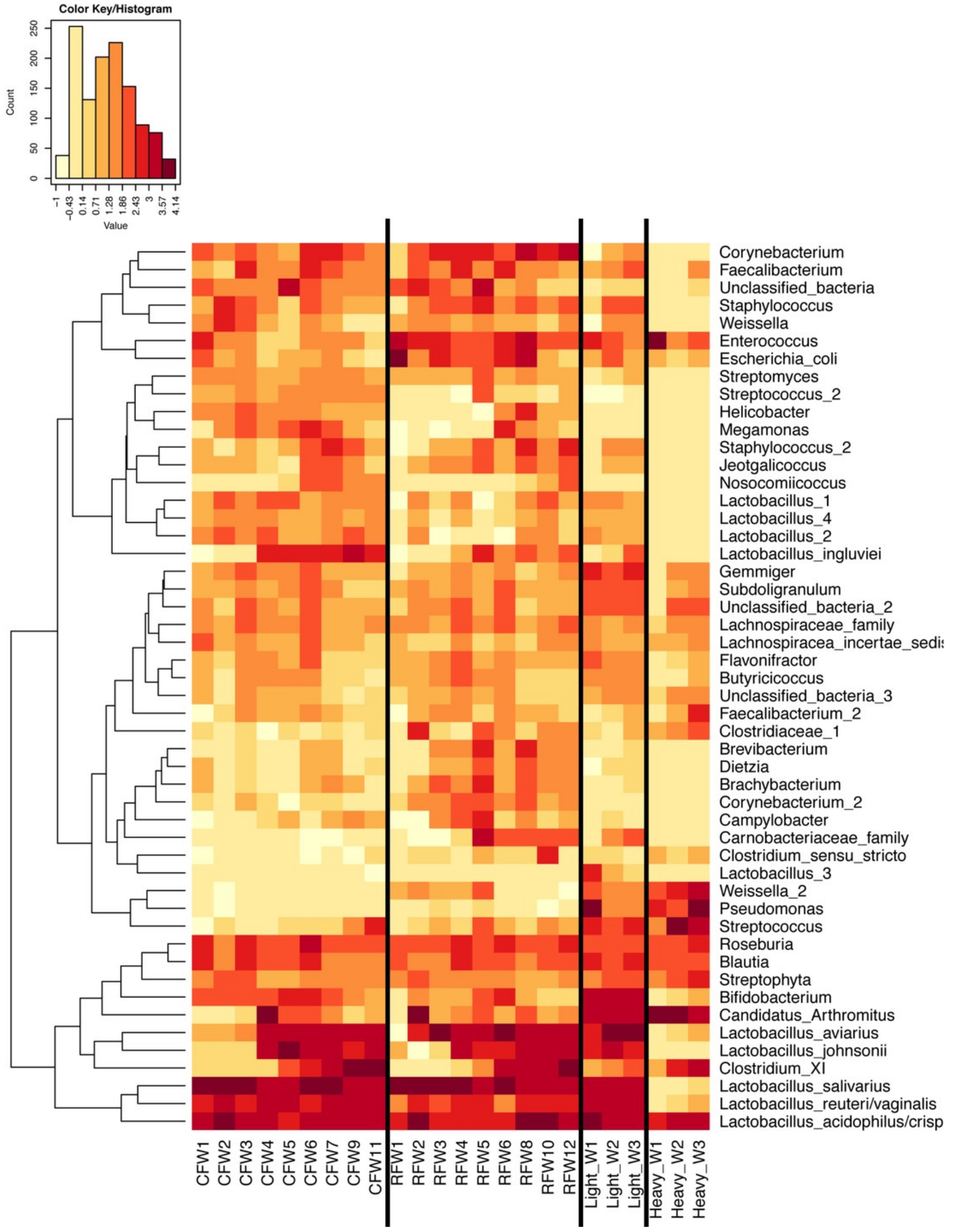


\section{Figure 7}

Heatmap of OTU abundance in individual birds.

Normalized counts for individual birds within each group and timepoint are depicted for the top 50 OTUs in this study. Heatmap is in $\log _{10}$ normalized counts. 

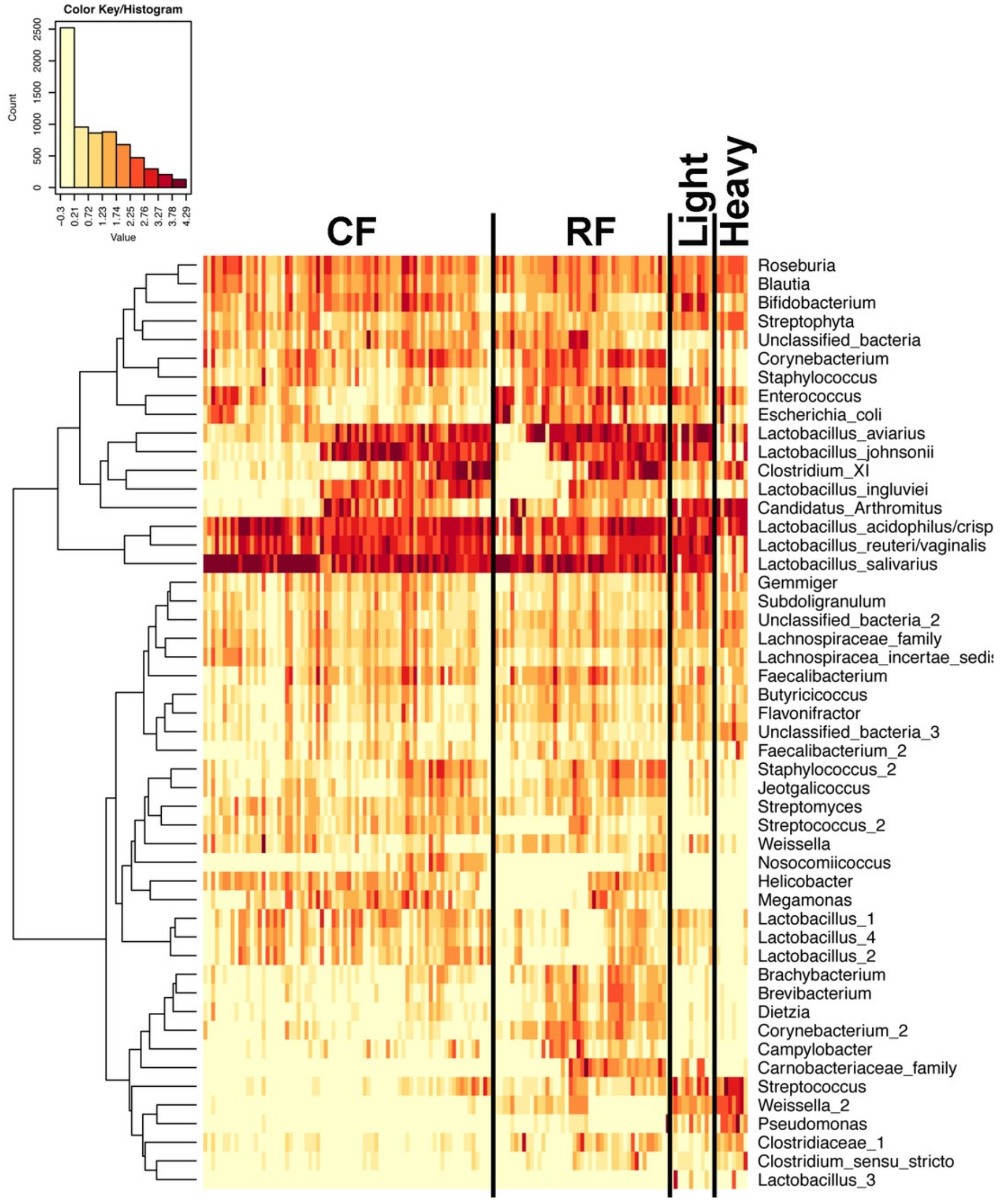


\section{Figure 8}

Principal coordinate analysis (PCOA) of samples of differing group and timepoint.

Dashed line indicates the approximate movement of turkeys from brooder to grow-out barns. Color is based on age of turkeys in weeks.

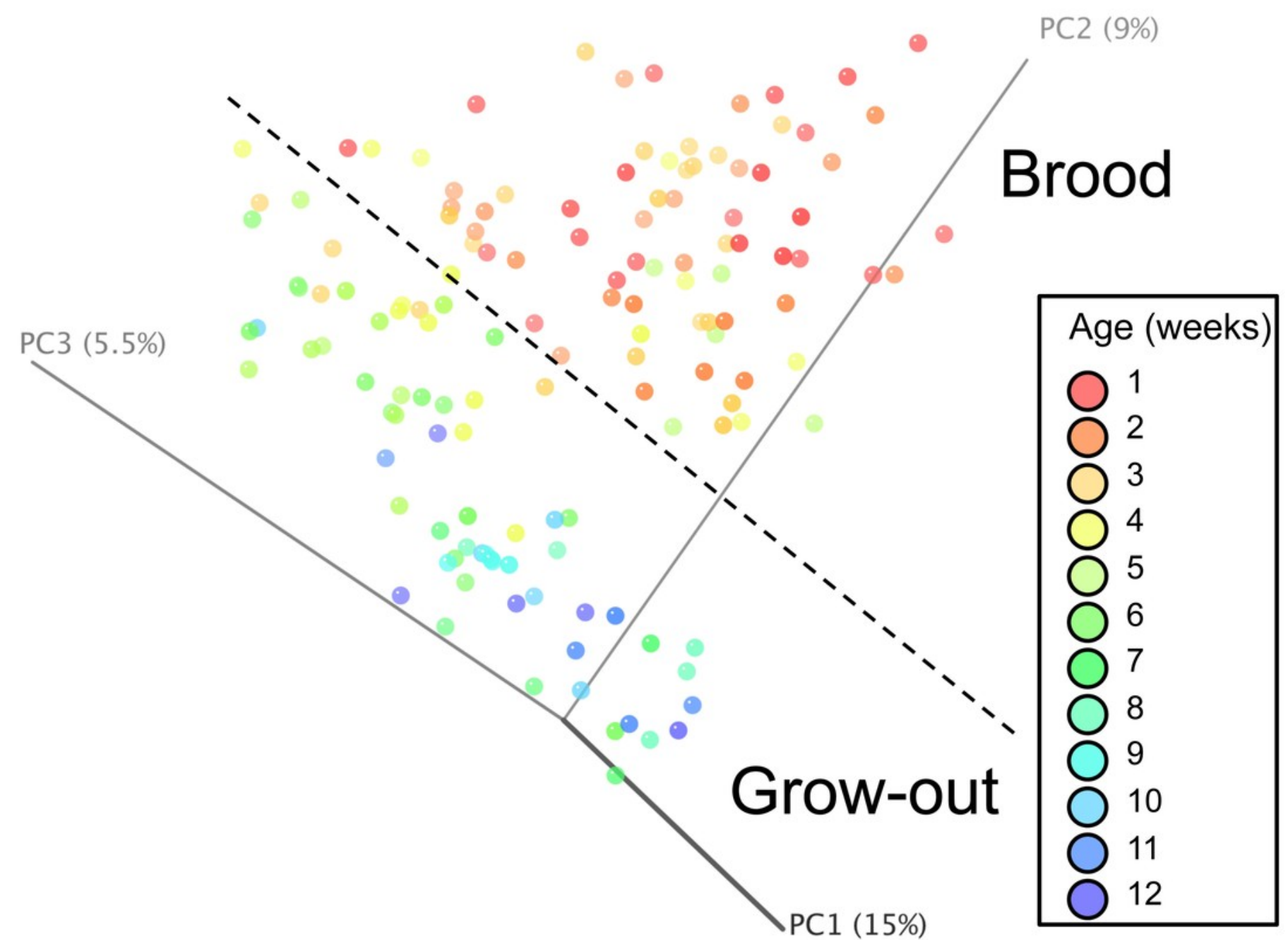




\section{Figure 9}

Cladogram depicting relationships between groups studied.

Cladogram was generated using two-way hierarchical clustering and Neighbor-Joining algorithm. Groups are designated as "CF" = commercial flock (experiment \#2), "RF" = research flock (experiment \#2), "Heavy" = flocks of heavier weights (experiment \#1), and "Light" = flocks of lighter weights (experiment \#1). 
Heavy W1

Heavy W3

Heavy W2

Light W1

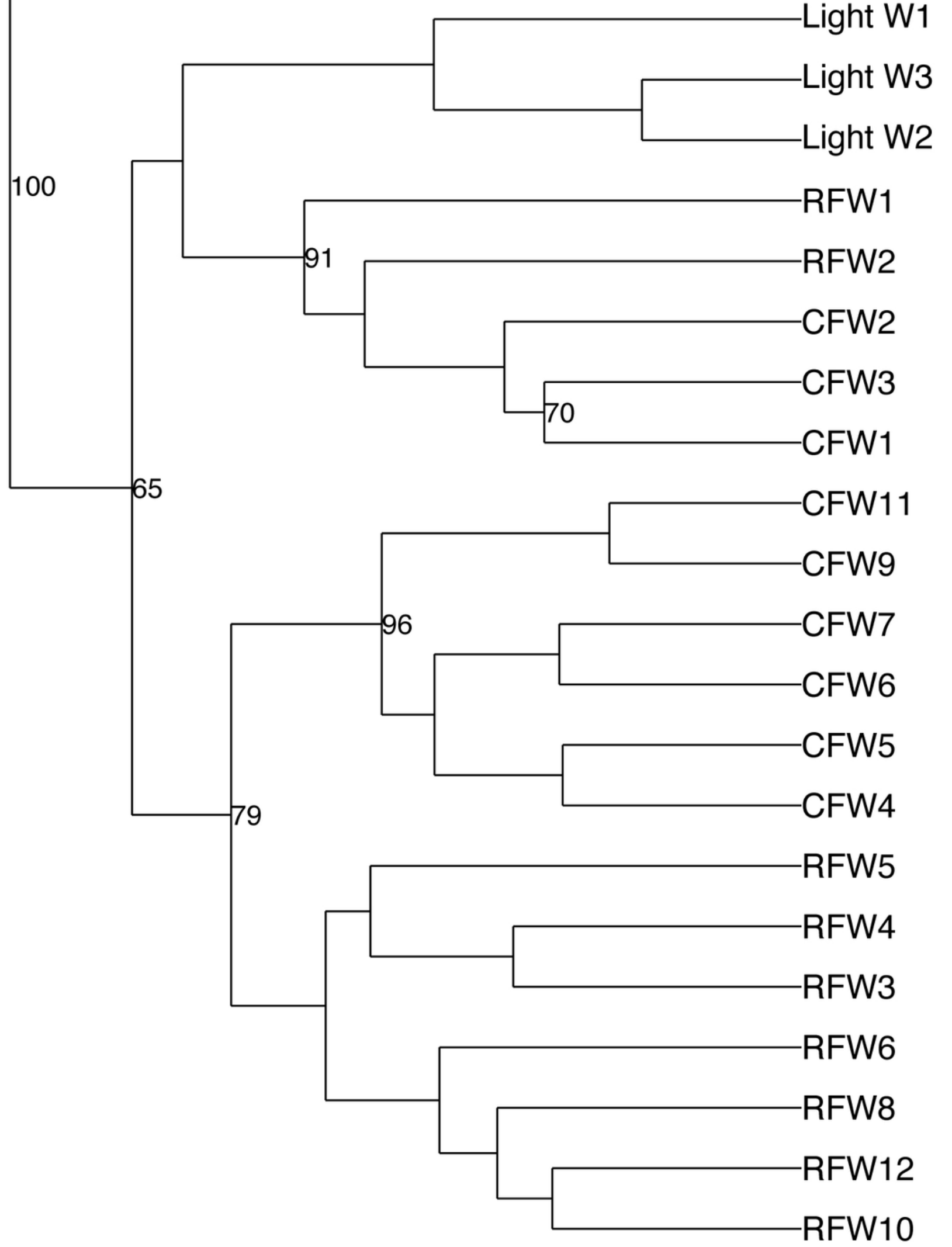




\section{Figure 10}

Temporal succession of bacteria in the turkey ileum.

Model is based upon OTUs identified as core in the dataset at each timepoint. Red-colored groups are present in weeks 1-2 and remain in the ileum through 7 weeks, green-colored groups emerge at 3-4 weeks, and blue-colored groups emerge at 5-7 weeks.

\begin{tabular}{|l|}
\hline \multicolumn{2}{|l|}{ Weeks 1-2 } \\
Lactobacillus dulbrueckii group \\
Enterococcus \\
Roseburia \\
Blautia \\
+ /- E. coli \\
\\
\hline Weeks 3-4 \\
\hline Lactobacillus salivarius \\
Lactobacillus dulbrueckii group \\
Lactobacillus aviarius \\
Lactobacillus johnsonii \\
Lactobacillus reuteri group \\
Roseburia \\
Bifidobacterim \\
Corynebacterium \\
Blautia
\end{tabular}

\section{Weeks 5-7}

Lactobacillus salivarius

Lactobacillus dulbrueckii group

Lactobacillus aviarius

Lactobacillus johnsonii

Clostridium Group XI

Lactobacillus reuteri group

Candidatus div. Arthromitus

Roseburia

Bifidobacterium

Corynebacterium

Blautia

Lactobacillus ingluviei 


\section{Table 1 (on next page)}

Samples analyzed in this study.

First letter in sample refers to flock sampled, with $\mathrm{F}=\mathrm{Light} /$ Heavy flocks, $\mathrm{CF}=$ commercial flock individual samples, and RF=research flock individual samples. "W" refers to week of age. 


\begin{tabular}{|c|c|c|c|c|c|}
\hline Sample & Flock type & $\begin{array}{l}\text { Birds } \\
(\mathrm{n})\end{array}$ & $\begin{array}{l}\text { Total filtered } \\
\text { sequences }\end{array}$ & $\begin{array}{l}\text { Total rarefied } \\
\text { sequences }\end{array}$ & Description \\
\hline F1W1 & Commercial & 40 & 54,868 & 20,000 & Pooled Light commercial flock \\
\hline F1W2 & Commercial & 40 & 96,067 & 20,000 & Pooled Light commercial flock \\
\hline F1W3 & Commercial & 40 & 46,694 & 20,000 & Pooled Light commercial flock \\
\hline F2W1 & Commercial & 40 & 35,379 & 20,000 & Pooled Light commercial flock \\
\hline F2W2 & Commercial & 40 & 42,313 & 20,000 & Pooled Light commercial flock \\
\hline F2W3 & Commercial & 40 & 43,083 & 20,000 & Pooled Light commercial flock \\
\hline F4W1 & Commercial & 40 & 19,651 & 20,000 & Pooled Light commercial flock \\
\hline F4W2 & Commercial & 20 & 20,472 & 20,000 & Pooled Light commercial flock \\
\hline F4W3 & Commercial & 40 & 47,365 & 20,000 & Pooled Light commercial flock \\
\hline F5W1 & Research & 40 & 62,525 & 20,000 & Pooled Heavy research flock \\
\hline F5W2 & Research & 20 & 7,870 & 7,870 & Pooled Heavy research flock \\
\hline F5W3 & Research & 20 & 21,258 & 20,000 & Pooled Heavy research flock \\
\hline F6W1 & Research & 40 & 41,978 & 20,000 & Pooled Heavy research flock \\
\hline F6W2 & Research & 40 & 56,625 & 20,000 & Pooled Heavy research flock \\
\hline F6W3 & Research & 40 & 42,878 & 20,000 & Pooled Heavy research flock \\
\hline F7W1 & Commercial & 20 & 19,877 & 20,000 & Pooled Light commercial flock \\
\hline F7W2 & Commercial & 40 & 39,240 & 20,000 & Pooled Light commercial flock \\
\hline F7W3 & Commercial & 20 & 14,785 & 20,000 & Pooled Light commercial flock \\
\hline F8W1 & Commercial & 40 & 49,918 & 20,000 & Pooled Heavy research flock \\
\hline F8W2 & Commercial & 40 & 85,002 & 20,000 & Pooled Heavy research flock \\
\hline F8W3 & Commercial & 20 & 8,855 & 8,855 & Pooled Heavy research flock \\
\hline RFW1 (1-5) & Research & 5 & 365,704 & 100,000 & Individuals from research flock \\
\hline RFW2 (1-5) & Research & 5 & 465,709 & 100,000 & Individuals from research flock \\
\hline RFW3 (1-5) & Research & 5 & 394,010 & 100,000 & Individuals from research flock \\
\hline RFW4 (1-5) & Research & 5 & 400,081 & 100,000 & Individuals from research flock \\
\hline RFW5 (1-5) & Research & 5 & 387,436 & 100,000 & Individuals from research flock \\
\hline RFW6 (1-5) & Research & 5 & 456,102 & 100,000 & Individuals from research flock \\
\hline RFW8 (1-5) & Research & 5 & 514,068 & 100,000 & Individuals from research flock \\
\hline RFW10 (1-5) & Research & 5 & 283,898 & 100,000 & Individuals from research flock \\
\hline RFW12 (1-5) & Research & 5 & $2,449,503$ & 100,000 & Individuals from research flock \\
\hline CFW1 (1-10) & Commercial & 10 & $1,691,968$ & 200,000 & Individuals from commercial flock \\
\hline CFW2 (1-10) & Commercial & 10 & $1,622,891$ & 200,000 & Individuals from commercial flock \\
\hline CFW3 (1-10) & Commercial & 10 & $1,701,982$ & 200,000 & Individuals from commercial flock \\
\hline CFW4 (1-10) & Commercial & 10 & $1,247,984$ & 200,000 & Individuals from commercial flock \\
\hline CFW5 (1-10) & Commercial & 10 & $1,723,505$ & 200,000 & Individuals from commercial flock \\
\hline CFW6 (1-9) & Commercial & 9 & $1,673,269$ & 180,000 & Individuals from commercial flock \\
\hline CFW7 (1-5) & Commercial & 5 & 635,010 & 100,000 & Individuals from commercial flock \\
\hline CFW9 (1-5) & Commercial & 5 & 766,290 & 100,000 & Individuals from commercial flock \\
\hline \multirow[t]{2}{*}{ CFW11 (1-5) } & Commercial & 5 & 481,758 & 100,000 & Individuals from commercial flock \\
\hline & Total & 834 & $18,117,871$ & $2,776,725$ & \\
\hline
\end{tabular}

